\title{
Exosomal myeloperoxidase as a biomarker of deep venous thrombosis
}

\author{
Yafei Han ${ }^{1}$, Xiaochun Bai ${ }^{2}$, Xinjia Wang ${ }^{1,3}$ \\ ${ }^{1}$ Department of Spine Surgery, the Second Affiliated Hospital, Shantou University Medical College, Shantou, China; ${ }^{2}$ Department of Cell Biology, \\ School of Basic Medical Sciences, Southern Medical University, Guangzhou, China; ${ }^{3}$ Department of Orthopedic, Affiliated Cancer Hospital, Shantou \\ University Medical College, Shantou, China \\ Contributions: (I) Conception and design: X Wang; (II) Administrative support: X Wang; (III) Provision of study materials or patients: X Wang, X Bai; \\ (IV) Collection and assembly of data: Y Han; (V) Data analysis and interpretation: Y Han, X Wang; (VI) Manuscript writing: All authors; (VII) Final \\ approval of manuscript: All authors. \\ Correspondence to: Xinjia Wang. The Second Affiliated Hospital, Shantou University Medical College, DongXia North Road, Shantou 515041, \\ China; Department of Orthopedic, Affiliated Cancer Hospital, Shantou University Medical College, RaoPing Road, Shantou 515041, China. \\ Email: xj.wang2000@163.com.
}

Background: Deep vein thrombosis (DVT) often occurs following major orthopedic surgery. In this study, we investigated specific exosomal proteins as potential diagnostic biomarkers of DVT.

Methods: Proteomic analysis of exosomes from four DVT patients and healthy controls $(\mathrm{n}=4)$ was performed by mass spectrometry. The model animals were evaluated at 1 inferior vena cava ligation [(IVCL)-1D], 3 (IVCL-3D), and 7 (IVCL-7D) days after IVCL. Endothelial cells in the thrombus segment were examined using terminal deoxynucleotidyl transferase dUTP nick end labeling (TUNEL) assays and hematoxylin and eosin (HE) staining. Myeloperoxidase (MPO) expression in the damaged vessel was detected by immunofluorescence staining. Exosomes were co-cultured with human umbilical vein endothelial cells (HUVECs) and cell proliferation was estimated using Cell Counting Kit-8 (CCK-8) assays.

Results: A total of 78 differentially expressed proteins (DEPs; 38 downregulated and 40 upregulated) were identified in the DVT group. In the rat DVT model, endothelial cells were damaged continuously after thrombosis, with the most serious injury in the IVCL-3D group, after which signs of endothelial repair were apparent. The IVCL-1D group showed the highest levels of vascular endothelial cell apoptosis and MPO increased sharply in the IVCL-1D and IVCL-3D groups, but had almost disappeared in the IVCL-7D group. In co-culture, plasma exosomes isolated from DVT model rats were efficiently absorbed by HUVECs, with markedly lower HUVECs growth and higher levels of apoptosis in the IVCL-1D and IVCL-3D groups compared with the control group.

Conclusions: Our findings suggest that exosomes may be involved in endothelial cell injury during DVT. The exosomal protein MPO is a potential biomarker of early stage DVT.

Keywords: Exosomes; deep vein thrombosis (DVT); myeloperoxidase; proteomic analysis

Submitted Oct 08, 2021. Accepted for publication Dec 08, 2021.

doi: $10.21037 / \mathrm{atm}-21-5583$

View this article at: https://dx.doi.org/10.21037/atm-21-5583

\section{Introduction}

Deep venous thrombosis (DVT) is a common complication following major orthopedic surgery such as hip, knee replacement, and spinal surgery and can cause death due to secondary pulmonary embolism. At present, the diagnosis of DVT depends mainly on vascular ultrasound and digital subtraction angiography technology. Although both methods have good diagnostic value, the former cannot diagnose intra-peritoneal venous embolism, and the latter is highly invasive and costly. 
Currently, there are no definitive laboratory indicators and detection methods for early diagnosis and prediction of DVT in clinic. Most doctors routinely use anticoagulants for patients undergoing major orthopedic surgery of the lower extremities; this is not only an economic expense, but can also induce severe complications such as intracranial bleeding (1). Therefore, definitive laboratory indicators for early diagnosis of DVT are urgently required.

Recent studies have indicated the potential of exosomes as diagnostic markers for some diseases, likely providing new ideas for DVT biomarkers. Exosomes contain unique microRNAs (miRNAs) and proteins, which not only function in substance transport and signal transmission, but also protect the substances contained within from degradation (2-4). Some unique miRNAs and proteins are more highly expressed in exosomes than in plasma. Studies have shown that exosomes can initiate endogenous and exogenous coagulation pathways by transmitting different signals, and play an important role in blood coagulation. Furthermore, there is accumulating evidence that exosomes play an important role in coagulation in trauma, tumors, and vascular sclerosis $(5,6)$.

Exosomes can affect vascular endothelial cell function as well as platelet activation and aggregation, increasing blood coagulability (7). Vascular endothelial cells play an important role in the stabilization of blood flow, and procoagulant factors secreted by endothelial cells directly and quickly participate in coagulation and thrombosis (8). Cytokines produced by endothelial cells after stimulation can induce monocytes to adhere to endothelial cells and stimulate platelet activating factors to participate in thrombosis (9). In this study, we explored potential exosomal biomarkers in the blood of DVT patients and investigated the effect of exosomes on vascular endothelial cells in cell culture and animal models. Compared with past experiments exploring then role of exosomes in the occurrence of DVT, exosomes were collected from human blood for proteomic analysis to obtain different target proteins in this experiment. Humanderived protein data makes it more clinically meaningful. We present the following article in accordance with the ARRIVE reporting checklist (available at https://dx.doi. org/10.21037/atm-21-5583).

\section{Methods}

\section{Collection of blood in DVT patients}

Ethylenediamine tetraacetic acid (EDTA)-anticoagulant venous blood was collected from 4 DVT patients (two males and two females; mean age, $56 \pm 15$ years) after total hip arthroplasty (THA) and 4 healthy volunteers (two males and two females; mean age, $56.5 \pm 12.5$ years). Blood samples were centrifuged at $3,000 \times \mathrm{g}$ for $10 \mathrm{~min}$ at $4{ }^{\circ} \mathrm{C}$ to remove cells. The plasma was collected and immediately frozen in liquid nitrogen for $15 \mathrm{~min}$, then $250 \mu \mathrm{L}$ aliquots were stored at $-80{ }^{\circ} \mathrm{C}$ prior to analysis. All procedures performed in this study involving human participants were in accordance with the Declaration of Helsinki (as revised in 2013). The study was approved by the Ethics Committee of the Second Affiliated Hospital of Shantou University Medical College (China) (No. 2019-35) and informed consent was taken from all the patients.

\section{Isolation and evaluation of exosomes}

The plasma sample was centrifuged at 20,000 $\times \mathrm{g}$ for $30 \mathrm{~min}$ at $4{ }^{\circ} \mathrm{C}$. The supernatant was then centrifuged at $100,000 \times \mathrm{g}$ for $70 \mathrm{~min}$ at $4{ }^{\circ} \mathrm{C}$. The resulting supernatant was discarded and the pellets were washed in a large volume of phosphatebuffered saline (PBS) and centrifuged at 100,000 $\times \mathrm{g}$ for $70 \mathrm{~min}$ at $4{ }^{\circ} \mathrm{C}$. The resulting pellet was collected and resuspended in PBS. After separation, the ultrastructure, concentration, and size distribution of exosomes were analyzed by electron microscopy and nanoparticle tracking analysis (NTA).

\section{Transmission electron microscopy}

The purified plasma exosomes were resuspended in PBS, dropped into the transmission electron microscopy (TEM) grid, which was switched to the absorb mode for $10 \mathrm{~min}$, and negatively stained with $2 \%$ phosphotungstic acid $(\mathrm{pH}$ 6.8) for $5 \mathrm{~min}$. After air-drying under an incandescent lamp, the plasma exosomes were examined under an electron microscope (Hitachi-H7650, Tokyo, Japan) at $120 \mathrm{kV}$.

\section{NTA}

The size distribution and number of isolated exosomes were examined with the Nanosight N3000 system (Malvern Panalytical, Malvern, UK) and the data were analyzed using NTA 3.2 Dev Build 3.2.16 (Malvern, UK).

\section{Proteomic analysis of exosomes}

Total proteins were extracted from plasma exosomes using radioimmunoprecipitation (RIPA) lysis buffer (BioSharp, Hefei, China), and the concentration was determined using the Pierce bicinchoninic acid (BCA) protein assay 
kit (Thermo Fisher Scientific, Rockford, IL, USA). Equal amounts of each protein sample were separated by $10 \%$ sodium dodecyl sulfate-polyacrylamide gel electrophoresis (SDS-PAGE) and transferred to a polyvinylidene fluoride (PVDF) membrane before incubation overnight with primary antibodies against CD63 and CD9 (1:1,500; Abcam, Cambridge, MA, USA), SHIP1, CDKN1B, and SOCS1 (1:800; Santa Cruz Biotechnology, Dallas, TX, USA), and $\beta$-actin and phosphorylated NF-KB p65 (1:1,000; Cell Signaling Technology, Beverly, MA, USA). Membranes were then incubated with horseradish peroxidase (HRP)conjugated secondary antibodies (1:4,000; Cell Signaling Technology). The protein bands were visualized using IMAGISOLANE LAS4000 Mini (GE Healthcare, Piscataway, NJ, USA). Image-Pro Plus 6.0 software (Mediacontrolnetics, Silver-Spring, MD, USA) was used to measure the scale values of protein bands, and the relative expression of the indicated proteins was normalized to the internal control $\beta$-actin.

\section{Studies of an animal model of venous thromboembolism}

Adult male Sprague-Dawley rats (8-10 weeks, 300-400 g) were purchased from the Experimental Animal Central of Southern Medical University (Guangzhou, China, 44002100019745). Experiments were performed under a project license (No. SUMC2019-244) granted by the Ethics Committee of the Second Affiliated Hospital of Shantou University Medical College (China), in compliance with guidelines of Experimental Animal Center of Medical College of Shantou University for the care and use of animals. A protocol was prepared before the study without registration. The experimental animals were anesthetized with $1 \%$ sodium pentobarbital $(300-400 \mu \mathrm{L} / 100 \mathrm{~g}$ body weight).

\section{Establishment of the rat model of DVT}

An inferior vena cava (IVC) ligation model was established as described previously (10). The IVC and all visible side branches (usually 2 or 3 ) were ligated with nonreactive 4-0 silk sutures. After 1, 3 and 7 days, the IVC and associated thrombus in each group were removed, weighed, and measured for thrombus length. The IVC and its branches in the control group rats were not ligated.

\section{Plasma exosome isolation and characterization}

Rat blood was collected into 5-mL EDTA anticoagulant tubes and plasma was obtained by centrifugation at 3,000 $\times \mathrm{g}$ for $10 \mathrm{~min}$ at $4{ }^{\circ} \mathrm{C}$ to remove cells and debris. The supernatant was then centrifuged at 20,000 $\times$ g for $30 \mathrm{~min}$ at $4{ }^{\circ} \mathrm{C}$ and the resulting supernatant was centrifuged at $100,000 \times \mathrm{g}$ for $70 \mathrm{~min}$ at $4{ }^{\circ} \mathrm{C}$. The supernatant was discarded and the pellets were washed in a large volume of PBS and centrifuged at $100,000 \times \mathrm{g}$ for $70 \mathrm{~min}$ at $4{ }^{\circ} \mathrm{C}$. Finally, the pellet was collected and resuspended in PBS. After separation, the ultrastructure, concentration, and size distribution of the plasma exosomes were analyzed by TEM and NTA.

\section{Hematoxylin and eosin staining}

Tissues were fixed with $4 \%$ paraformaldehyde, embedded in paraffin, and sectioned (thickness, $4 \mathrm{~mm}$ ) for dewaxing and dehydration. Hematoxylin and eosin (HE) staining was performed as previously described (11).

\section{Terminal deoxynucleotidyl transferase dUTP nick end labeling assays}

After the cells or tissues had been fixed with $4 \%$ paraformaldehyde and sectioned, in situ nick end labeling of nuclear DNA fragments was performed using a terminal deoxynucleotidyl transferase dUTP nick end labeling (TUNEL) apoptosis assay kit (Promega, Madison, WI, USA) according to the manufacturer's instructions. The samples were then analyzed under a laser scanning confocal microscope (Olympus, Japan).

\section{Western blot analysis}

Proteins were extracted from exosomes or tissues using RIPA lysis buffer (Biosharp, China) and concentrations were determined using the BCA protein assay kit (Absin, Shanghai, China). Equal amounts of protein samples were separated by $10 \%$ SDS-PAGE, transferred to a Polyvinylidene fluoride PVDF membrane, and incubated overnight with the following primary antibodies $(1: 1,000)$ : mouse monoclonal antibodies against glyceraldehyde 3-phosphate dehydrogenase (GAPDH; Proteintech, Wuhan, China) and CD63 (Abcam) and rabbit monoclonal antibodies against ALG 2-interacting protein X (Alix; Abcam), myeloperoxidase (MPO; Proteintech), and tumor susceptibility gene 101 (TSG101; Abcam). The membranes were then incubated with HRP-conjugated anti-rabbit or anti-mouse secondary antibodies (1:4,000; Invitrogen, Chicago, IL, USA) at room temperature for $1 \mathrm{~h}$. Protein bands were visualized using an OPTIMAX X-Ray Film 
Processor (PROTEC, Oberstenfeld, Germany).

\section{Immunofluorescence}

For immunofluorescence staining, tissues were fixed with $4 \%$ paraformaldehyde, and antigen repair was achieved by incubation with $0.25 \%$ citrate (ShiFeng Biology, Shanghai, China) for $16 \mathrm{~h}$ at $60{ }^{\circ} \mathrm{C}$. Sectioned tissues were blocked with goat serum at room temperature for $0.5 \mathrm{~h}$ before incubation overnight at $4{ }^{\circ} \mathrm{C}$ with the following primary antibodies (1:500): mouse monoclonal antibody against CD31 (Proteintech) and rabbit monoclonal antibody against MPO (Proteintech). Sections were incubated for $1 \mathrm{~h}$ at room temperature with fluorescently labeled anti-rabbit or anti-mouse secondary antibody (1:1,000, Invitrogen). Nuclei were stained with 4',6-diamidino-2-phenylindole (DAPI) for $10 \mathrm{~min}$. The images were captured under a laser scanning confocal microscope (Olympus).

\section{Uptake of exosomes by human umbilical vein endothelial cells}

Exosomes were fluorescently labeled using the PKH67 kit (Sigma-Aldrich, St. Louis, MO, USA) according to the manufacturer's instructions. After washing with PBS (Gibco, Grand Island, NY, USA), exosomes were centrifuged at $100,000 \times \mathrm{g}$ for $70 \mathrm{~min}$. Exosomes $(1 \mu \mathrm{g})$ were then cocultured with 100,000 human umbilical vein endothelial cells (HUVECs) for $4 \mathrm{~h}$ at $37{ }^{\circ} \mathrm{C}$ and $5 \% \mathrm{CO}_{2}$. The uptake of PKH67-labeled exosomes by HUVECs was analyzed under a laser scanning confocal microscope (Olympus).

\section{HUVECs culture}

We purchased HUVECs from American Type Culture Collection and cultured them in endothelial cell complete medium containing endothelial cell growth supplement (AllCells, Alameda, CA, USA) at $37{ }^{\circ} \mathrm{C}$ in a humidified atmosphere containing $5 \% \mathrm{CO}_{2}$.

\section{Cell Counting Kit-8 assay (CCK-8)}

The HUVECs were seeded in 96-well plates with 5,000 cells in each well. Exosomes $(0.05 \mu \mathrm{g})$ were added into each well and co-cultured with HUVECs for $72 \mathrm{~h}$. Subsequently, $10 \mu \mathrm{L}$ CCK- 8 solution was added to each well and the cells were incubated for $3 \mathrm{~h}$ at $37^{\circ} \mathrm{C}$. Absorbance was measured at $450 \mathrm{~nm}$ using a microplate reader (BioTek, Carlsbad, CA, USA) and data were analyzed using Gen 5 software (BioTek).

\section{Statistical analysis}

Experimental data were described as the mean \pm standard deviation, and a 2-tailed Student's $t$-test (GraphPad Prism software Version 5.0; San Diego, CA, USA) was used to assess statistical significance. A $P$ value $<0.05$ was considered to indicate statistical significance.

\section{Results}

Extraction and verification of exosomes from DVT patients

Exosomes were isolated by density gradient centrifugation from the blood of 4 DVT patients after THA and 4 healthy controls. Evaluation by TEM and NTA showed that the diameter of the isolated exosomes $(100-150 \mathrm{~nm})$ was consistent with the size of exosomes (Figure 1). Detection of the exosome marker ALG 2-interacting protein X (Alix) revealed that the content of exosomes in the DVT group was higher than that in the control group (Figure 1).

\section{Label-free quantitative proteomic analysis of exosomes in the plasma of orthopedic DVT patients}

A large number of differentially expressed exosomal proteins were distributed in the plasma of the DVT group compared with the control group (Figure 2). In total, 78 differentially expressed proteins (DEPs) were identified in the DVT group; 38 were downregulated and 40 were upregulated. Bioinformatics analysis revealed 5 significant DEPs (S100, MPO, aminopeptidase N, haptoglobin, and histone $\mathrm{H} 4$ ) related to thrombosis (Table 1).

\section{HE staining showed IVC vascular injury in an animal model of DVT}

Venous thrombosis was confirmed in (venous thromboembolism) VTE model rats. In the IVCL1D group, there was almost no gap between the venous thrombus and the vessel wall (Figure 3). By contrast, there was a visible gap between the blood vessel wall and the thrombus in the IVCL-3D and IVCL-7D groups. The blood vessel walls in the experimental groups were thinner than those in the control group. Among the experimental groups, the blood vessel walls were thinnest in the IVCL-3D group and thickest in the IVCL-7D group. The vessel walls were smooth and intact in the control group. Nevertheless, the intima of the vessel walls in each 

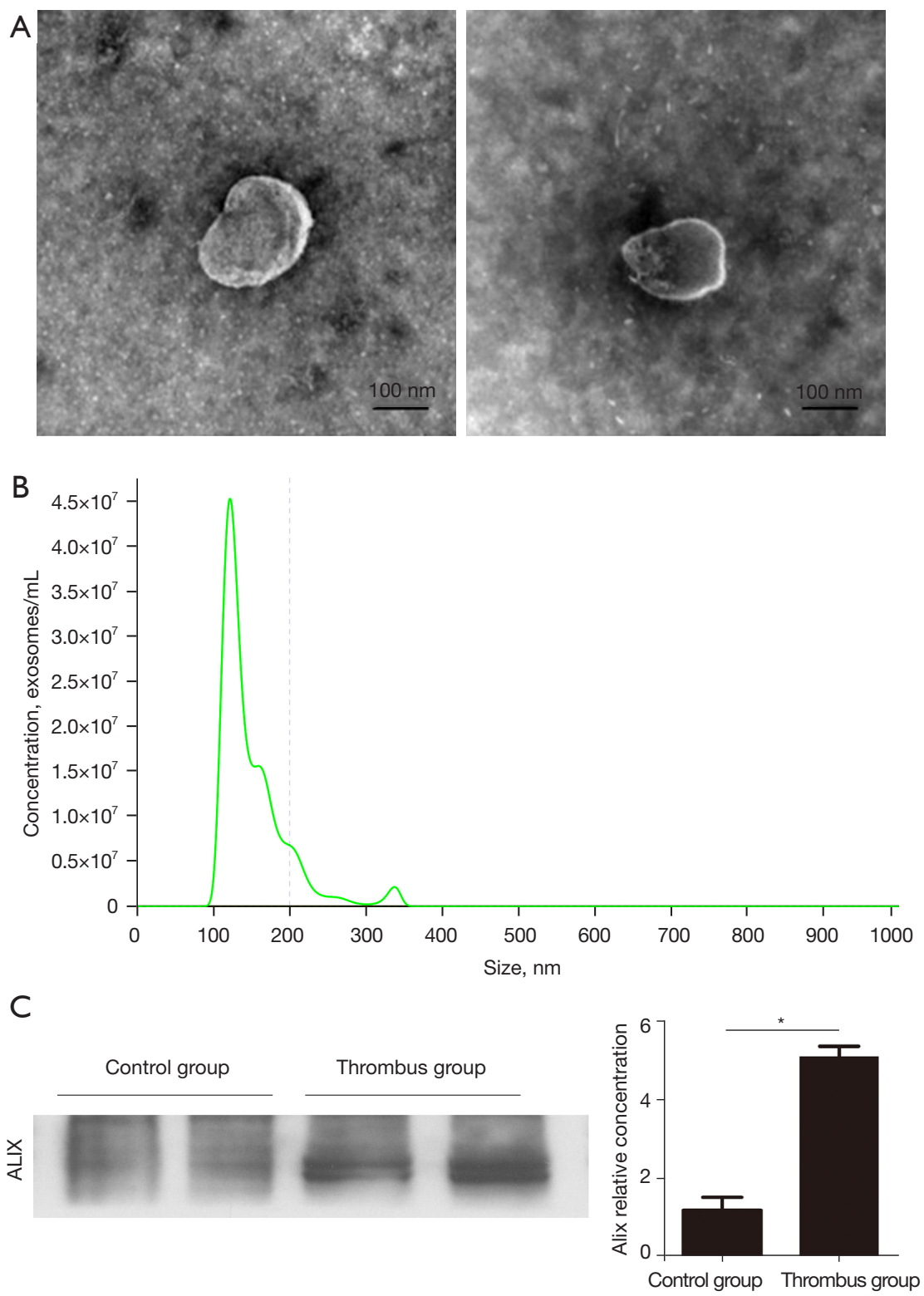

Figure 1 Identification of human plasma exosomes in the experimental group and the control group. (A) Transmission electron micrograph of exosomes $(\times 5,000 \mathrm{~nm})$. (B) Nanoparticle tracking analysis. Exosome diameters ranged from 100 to $150 \mathrm{~nm}$. (C) Higher expression of Alix was observed in the thrombus group compared with the control group. *, $\mathrm{P}<0.05$.

experimental group was damaged and the continuity was interrupted in the IVCL-3D group, while intima repair and thickening was observed in the IVCL-7D group. These results showed that endothelial cells are damaged continuously after thrombosis, with the most serious injury occurring on the third day after ligation, after which signs of endothelial repair were apparent.

\section{Detection of vascular endothelial cell apoptosis in animal models with thrombosis by TUNEL staining}

The TUNEL staining in VTE model rats showed low levels of apoptotic cells in the IVC of the control and IVCL-7D groups, with no marked difference between the 2 groups. Higher levels of TUNEL staining were detected 


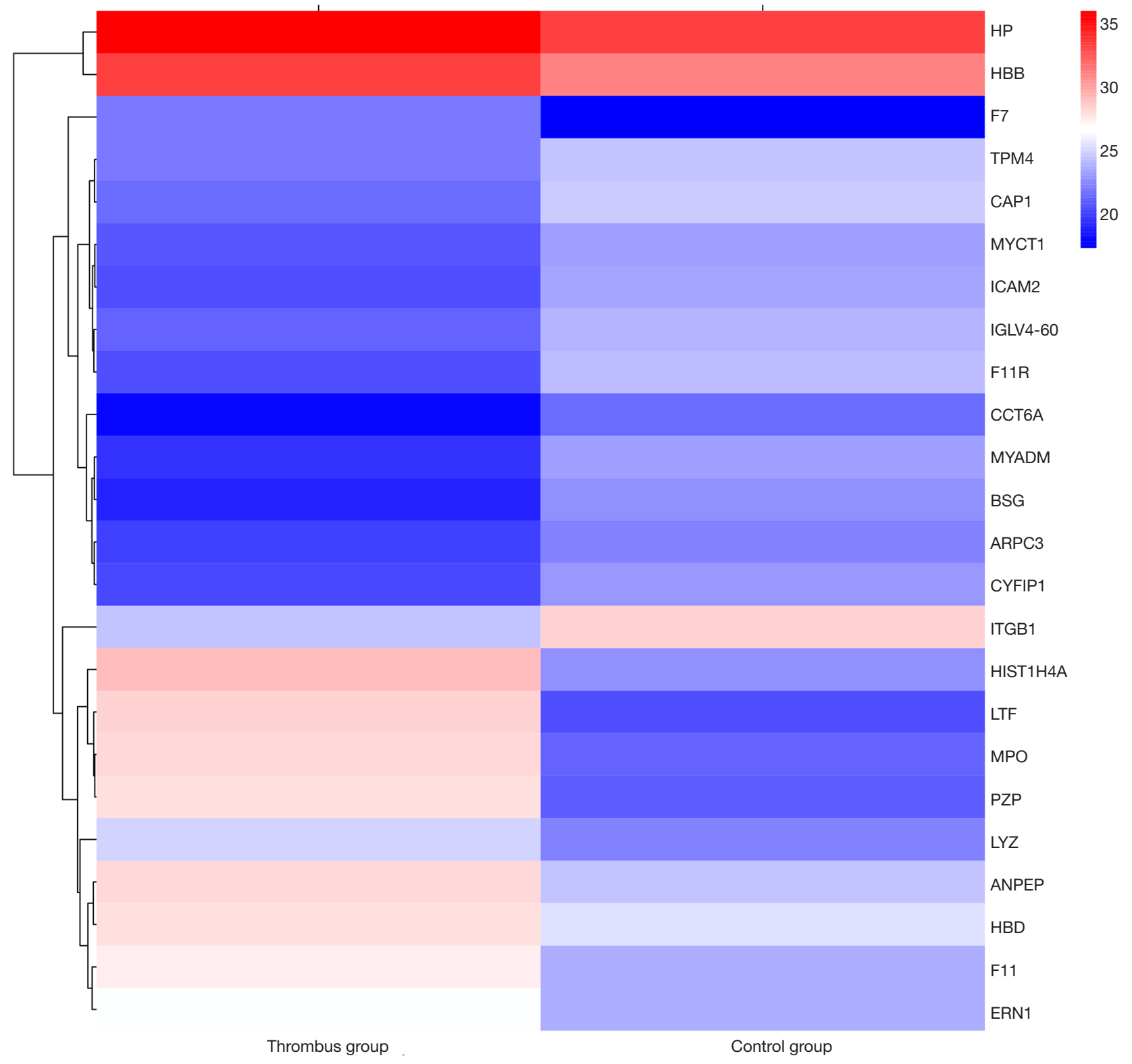

Figure 2 Proteomics analysis of plasma exosomes from patients with DVT and healthy individuals. Heat maps of 24 significantly differentially expressed exosomal proteins obtained from the plasma of participants in the thrombus and control groups. DV'T, deep vein thrombosis.

in the IVCL-1D group. The highest level of apoptosis was detected in the IVCL-3D group (Figure 3).

\section{Western blot analysis of MPO content}

Western blot analysis showed that, compared with the control group, MPO expression in vascular tissues increased sharply in the IVCL-1D and IVCL-3D groups, and decreased in the IVCL-7D group (Figure 4). Expression of the exosome markers CD63 and Alix was increased in the IVCL groups.

\section{Immunofluorescence staining of MPO distribution in the IVC of VTE model rats}

The distribution of MPO in blood vessels was detected 
Table 1 Differential expression of five exosome proteins in thrombosis group and control group $(\mathrm{P}<0.01)$

\begin{tabular}{lcccc}
\hline Protein name & DVT group & Healthy group & Fold change (DVT group/healthy group) & P value \\
\hline Protein S100-a-A9 & $213,744,522$ & $8,835,305.5$ & 24.19209183 & 0.000602 \\
Myeloperoxidase & $330,053,495.5$ & $2,103,409$ & 156.9136081 & 0.000075 \\
Aminopeptidase N & $290,852,279$ & $24,308,406$ & 11.96509055 & 0.004872 \\
Haptoglobin & $78,457,229,874$ & $12,762,601,692$ & 6.1474323 & 0.000071 \\
Histone H4 & $667,621,969$ & $6,234,318.5$ & 107.0882036 & 0.000762 \\
\hline
\end{tabular}

DVT, deep vein thrombosis.

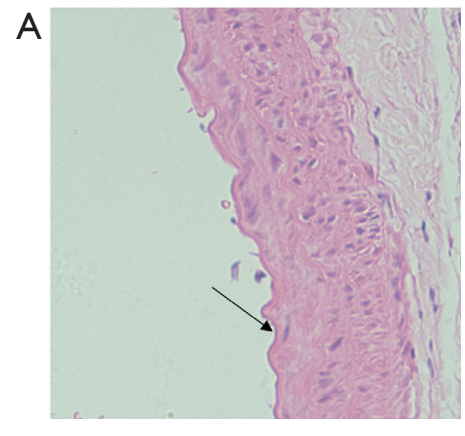

Control

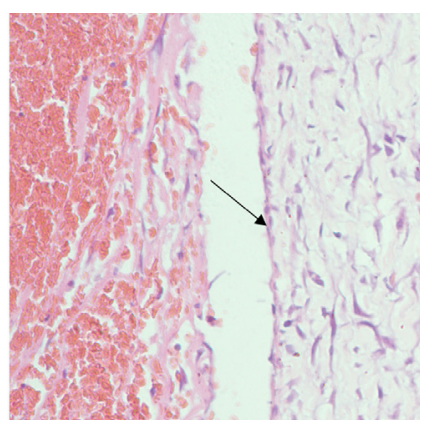

IVCL-3D

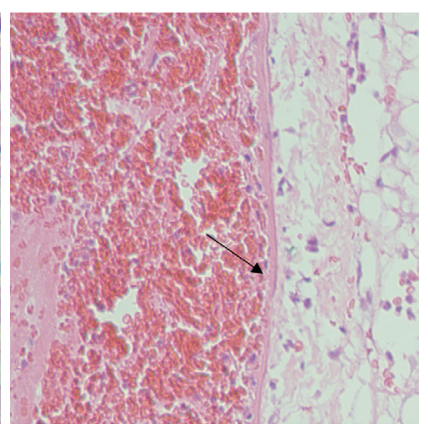

IVCL-1D

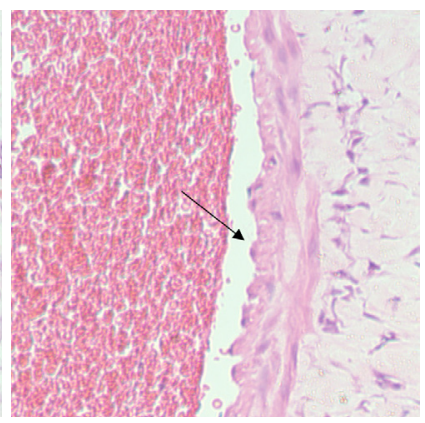

IVCL-7D

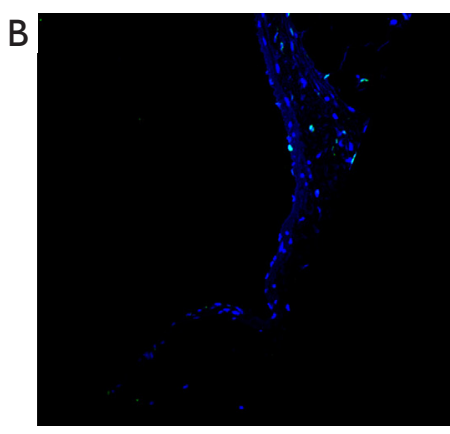

Control

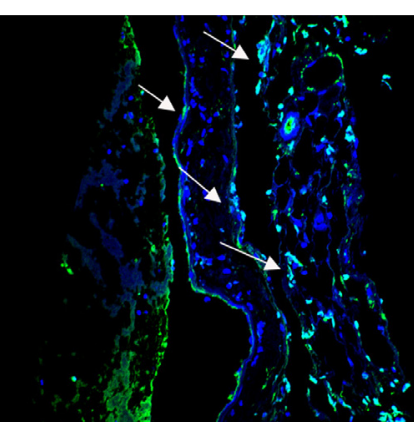

IVCL-3D

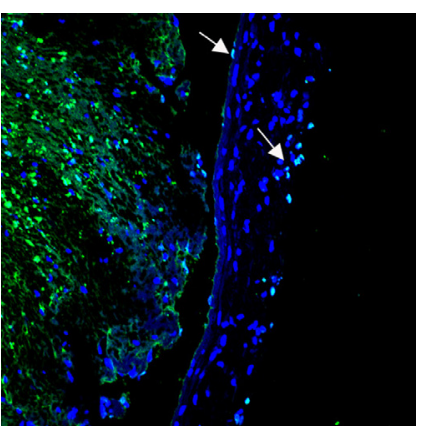

IVCL-1D

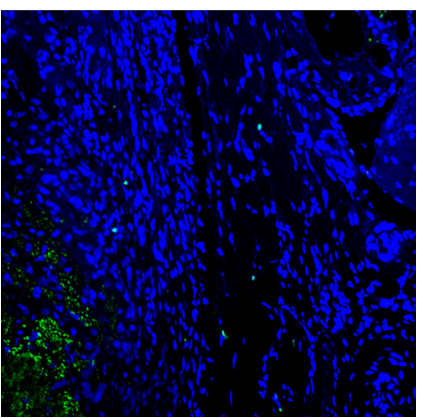

IVCL-7D

Figure 3 Images of inferior vena cava injury at different time points (1, 3, and 7 days) after ligation. (A) HE staining showing that the continuity and integrity of blood vessels were disrupted following inferior vena cava ligation, and the vessel wall was most severely damaged 3 days after ligation $(\times 40 \mu \mathrm{m})$. The black arrows indicate the morphology of the inferior vena cava vessel wall in rats treated with different methods. (B) TUNEL staining showing that venous blood vessel apoptosis was most obvious at 3 days after ligation ( $\times 40 \mu$ m). The white arrow indicates the apoptotic tissue in the inferior vena cava vessel wall of the rat. IVCL, inferior vena cava ligation; HE, hematoxylin and eosin; TUNEL, terminal deoxynucleotidyl transferase dUTP nick end labeling.

using immunofluorescence following IVC thrombosis (Figure 4). We performed DAPI staining to visualize the cell nuclei and CD31 was detected as a marker of endothelial cells. The content of MPO increased after venous thrombosis and was distributed mainly on the blood vessel wall in the IVCL-1D group, and mainly in the thrombus in the IVCL-3D group, while MPO expression had almost disappeared in the IVCL-7D group (Figure 4).

\section{MPO content in plasma exosomes isolated from VTE model rats increased following IVC thrombosis}

Exosomes were extracted and separated from the plasma of each group of animals. Exosomes were identified by 

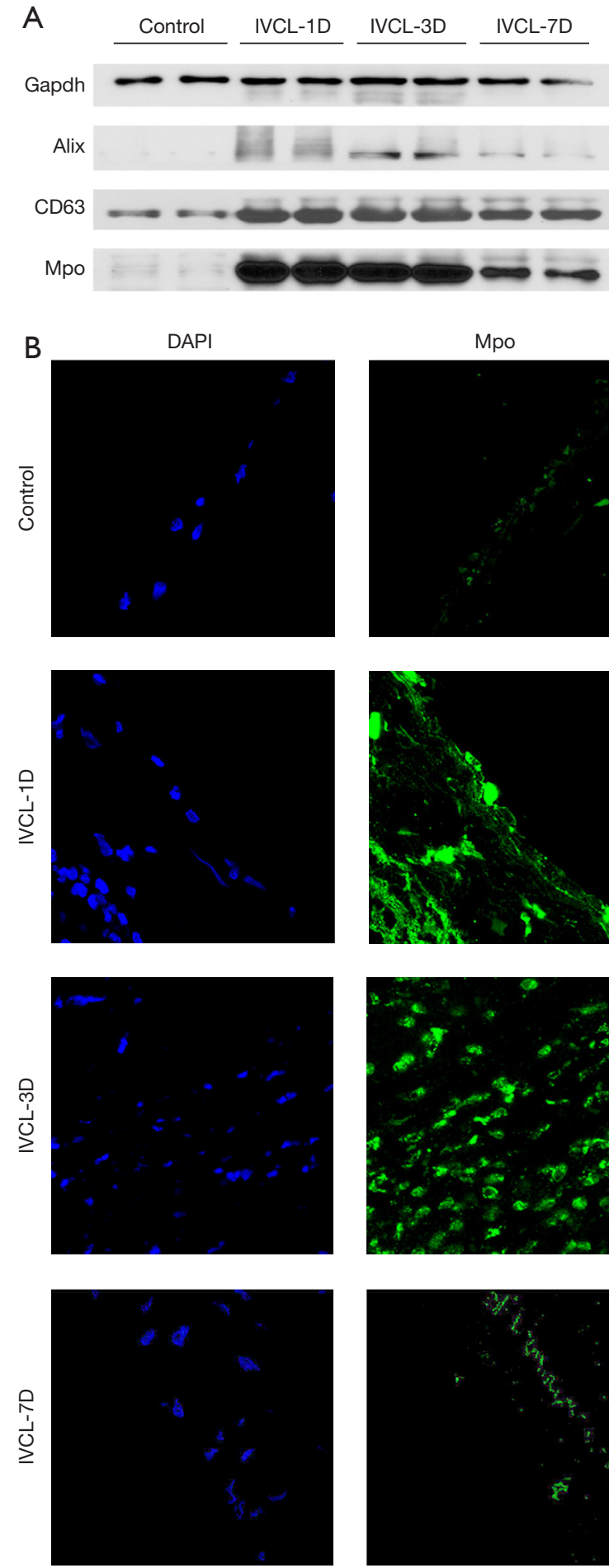

Mpo
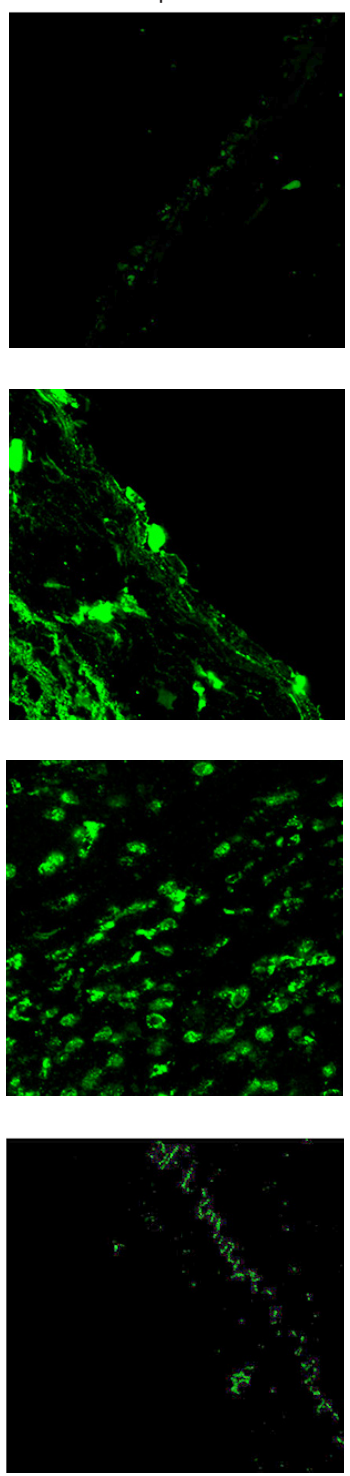

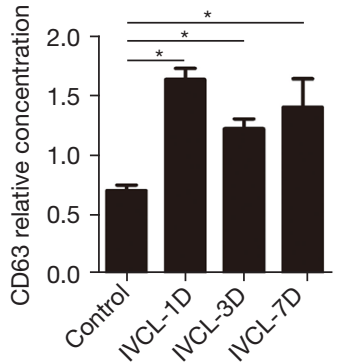

CD31
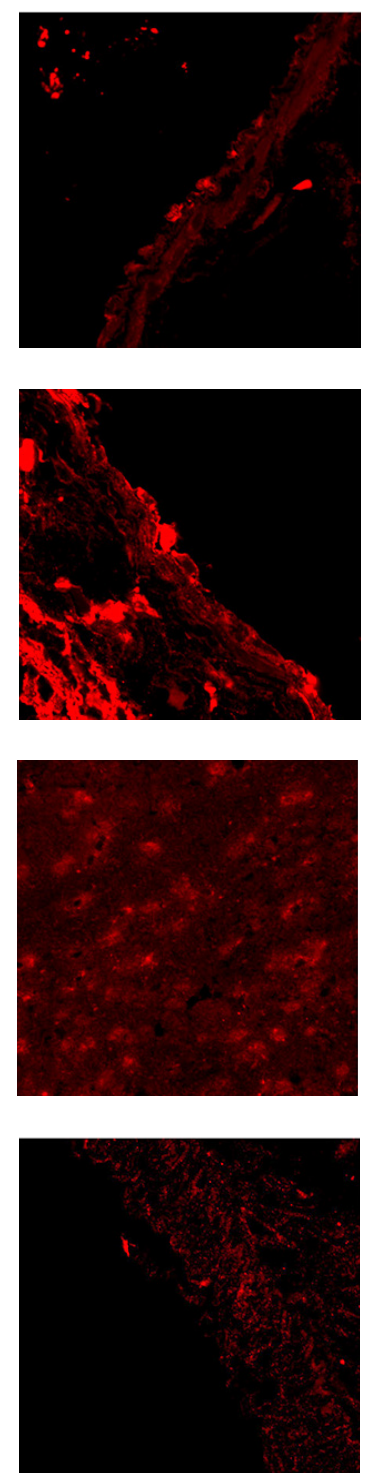

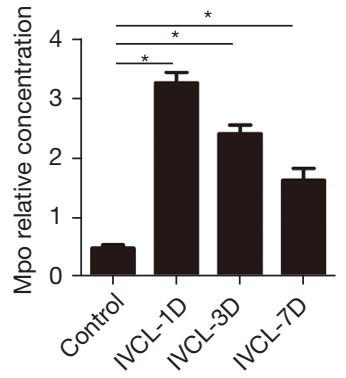

Merge
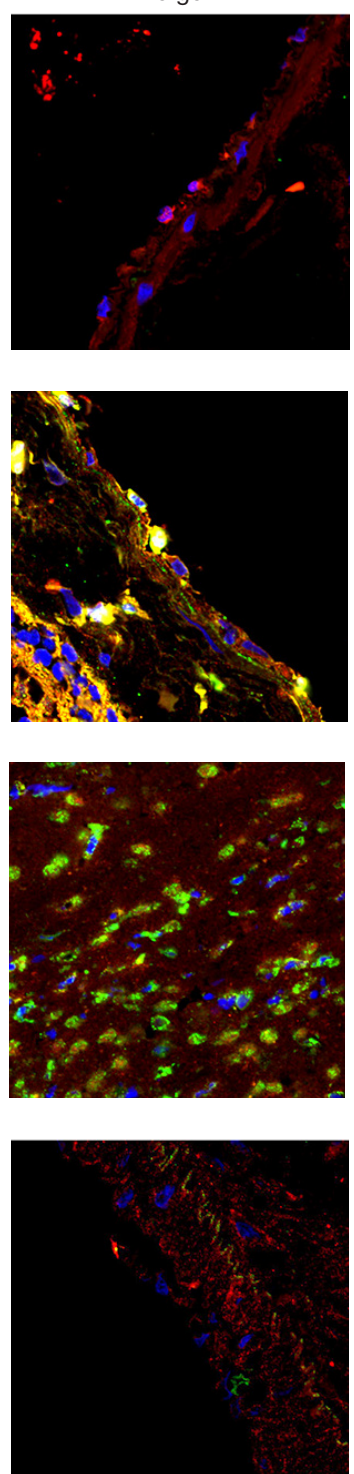

Figure 4 The distribution of MPO content in the inferior vena cava over time after inferior vena cava thrombosis. (A) Western blot analysis of MPO expression at different time points after venous thrombosis (1, 3, and 7 days). MPO was higher in the IVCL-1D and IVCL3D groups, but significantly lower in the IVCL-7D group. Expression of the exosomal markers CD63 and Alix increased after venous thrombosis. (B) Immunofluorescence analysis of the distribution of MPO in the inferior vena cava at different time points after thrombosis. MPO was mainly distributed in the blood vessel wall in the IVCL-1D group and in the emboli in the IVCL-3D group, while it had almost disappeared in the IVCL-7D group $(\times 50 \mu \mathrm{m})$. *, $\mathrm{P}<0.05$. DAPI, 4',6-diamidino-2-phenylindole; MPO, myeloperoxidase. 
A
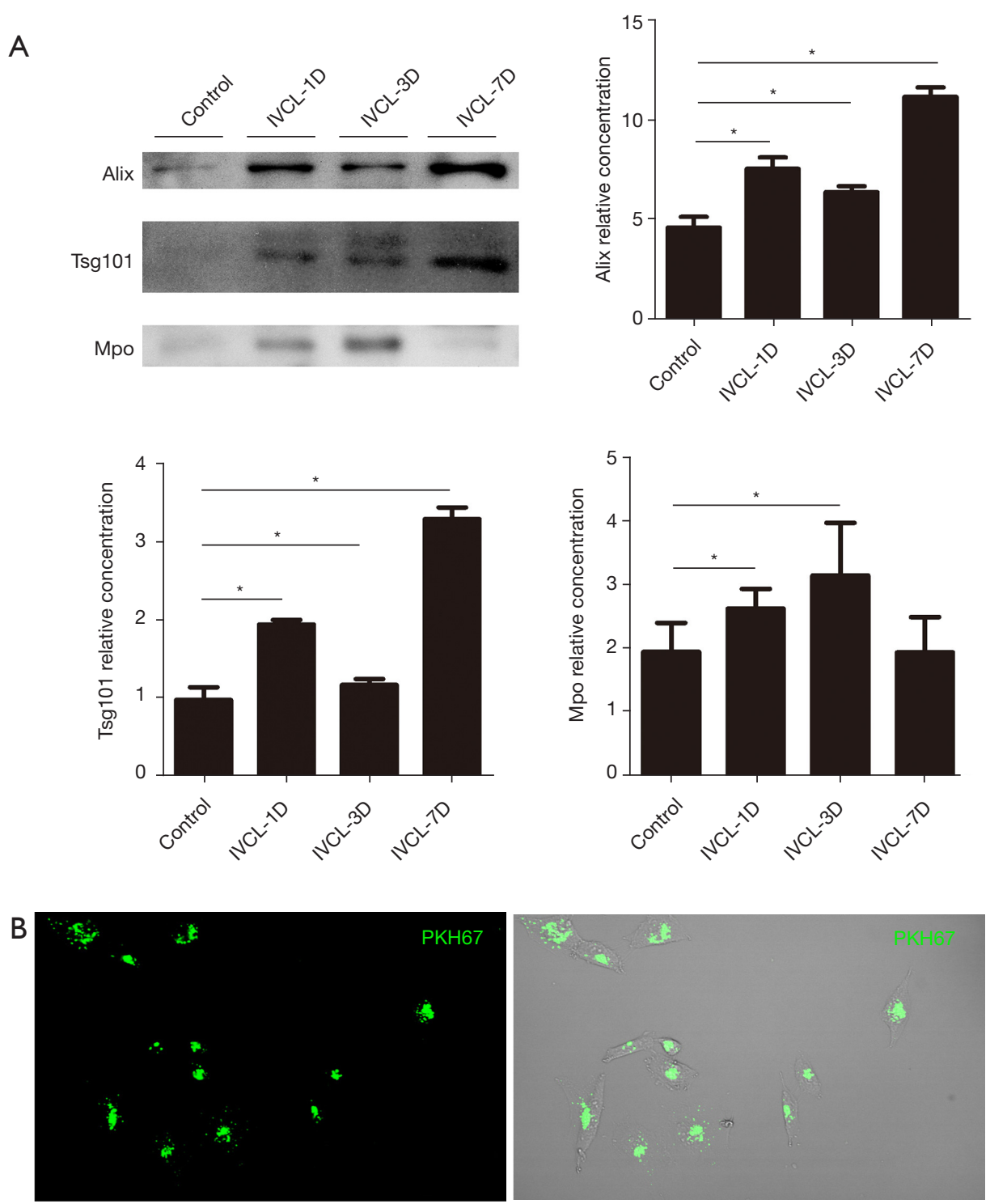

Figure 5 HUVECs uptake of plasma exosomes containing MPO. (A) Levels of CD63 and Alix in the IVCL-1D, IVCL-3D, and IVCL$7 \mathrm{D}$ groups was increased relative to levels of the control group. The content of MPO in the IVCL-1D and IVCL-3D groups increased significantly. (B) Exosomes labeled with green fluorescence were absorbed in large quantities by HUVECs. * $\mathrm{P}<0.05 . \times 200$ um. HUVECs, human umbilical vein endothelial cells; TSG101, tumor susceptibility gene 101; MPO, myeloperoxidase.

TEM and NTA, and the MPO content was measured by western blot analysis of Tsg 101 and the exosome marker Alix. Compared with the control group, the exosomal MPO content in the thrombus groups increased, with the highest MPO levels detected in the IVCL-3D group (Figure 5).

\section{HUVECs take up plasma exosomes}

The exosomes extracted from plasma were labeled with
PKH67 and co-cultured with HUVECs for $4 \mathrm{~h}$. As shown in Figure 5, a large quantity of exosomes was absorbed by HUVECs.

\section{MPO-containing plasma exosomes promote HUVECs injury and apoptosis}

Plasma exosomes isolated from VTE model rats were cocultured with HUVECs for $72 \mathrm{~h}$ and examined by light 
microscopy. As shown in Figure 6A, the proliferation of HUVECs co-cultured with plasma exosomes from the IVCL-1D and IVCL-3D groups was significantly poorer than those co-cultured with exosomes from the control and IVCL-7D groups. The HUVECs co-cultured with exosomes from the IVCL-3D group exhibited the lowest proliferation ability, with predominantly rounded cell morphology typical of naïve cells (Figure $6 B$ ). We conducted CCK-8 assays to detect the number of HUVECs in each group. The highest and lowest numbers of HUVECs were detected in the control and IVCL-3D groups, respectively (Figure 6). The TUNEL staining revealed visibly more apoptotic cells in the IVCL-1D and IVCL-3D groups than in the control and IVCL-7D groups (Figure 6C).

\section{Discussion}

A recent study found that soluble coagulation factors are as important as endothelial cell damage, platelet adhesion, aggregation, and release in venous thrombosis (12). The condition of DVT is a complex inflammatory response process involving a large number of inflammatory cells and inflammatory factors (13). Abnormal expression of inflammatory factors can cause excessive apoptosis of endothelial cells and lead to vascular wall damage (14). In the present study, we confirmed that impaired endothelial cell growth combined with increased apoptosis occur following venous thrombosis. In clinical practice, some patients show no symptoms in early stage DVT, which is called occult thrombus. Early diagnosis of clinical occult venous thrombosis not only reduces late complications of venous thrombosis but also reduces bleeding events in patients caused by the use of anticoagulant drugs. In recent studies, potential novel biomarkers for improving the diagnosis of DVT include P-selectin, factor VIII, Soluble platelet endothelial cell adhesion molecule 1 (sPECAM-1), Activated protein C-protein C inhibitor (APC-PCI) (15-18). By studying the role of exosomes during venous thrombosis in this study, we identified exosomal MPO as a potential biomarker for early diagnosis of DVT.

DVT and related complications impose a heavy burden on the national health-care system (19). Therefore, DVT has drawn much attention and has been well studied in the past few years. Some previous studies have elaborated that DVT is correlated with advanced age, obesity, smoking, trauma, major surgery, high blood pressure (HBP), blood transfusion, immobilization, hospitalization, neurological deficits, hypercoagulability, malignancy, D-dimer level, and oral contraceptive use (20-22). However, the lack of clinically accurate indicators to predict the occurrence of DVT has always troubled clinicians. The results of this study provide a novel early diagnostic biomarkers for highrisk patients developing DVT based on exosomal MPO in circulation.

Mainly derived from neutrophils and is a lysosomal protein that occurs in azophilic granules (23), MPO has well known properties of promoting inflammatory responses and oxidative stress (24). A host of inflammatory cells and inflammatory factors such as tumor necrosis factor alpha and interleukin 1 are involved in the inflammatory response (25). In arterial thrombosis, a large amount of MPO is found in atherosclerotic plaques (26). Fecal MPO has been proposed as a diagnostic biomarker of inflammatory bowel disease (27). IN addition, MPO mediates inflammatory responses and oxidative stress that induce cardiovascular disease (28). Thus, MPO may play an important role in DVT. In this study, we investigated the role of MPO in the pathogenesis of orthopedic surgery patients with DVT. We found that MPO was abundantly distributed in the vessel walls and emboli within 1 to 3 days of DVT. In exosomes isolated from the plasma of DVT model rats, MPO remained at a high concentration within 1 to 3 days of DVT, but decreased significantly after 7 days. Interestingly, exosomes isolated from the plasma of model rats at 3 days after DVT induced damage to vascular endothelial cells and promoted apoptosis. However, exosomes isolated from the plasma of model rats at 7 days after DVT did not induce endothelial cell injury or apoptosis. Based on these observations of the synchronous relationship between the MPO content of plasma exosomes and the damage to endothelial cells induced by these exosomes, we speculated that the increased levels of exosomal MPO might indicate damage to endothelial cells in early stage DVT. Thus, exosomal MPO may be a potential biomarker for early diagnosis of DVT.

The functions of exosomes are influenced by external stimuli. Under different environmental stimuli, exosomes can play important roles throughout the body by transporting different substances. The microparticles in patients with $\beta$-thalassemia can activate vascular endothelial cells and increase the adhesion of monocytes, thereby increasing the risk of thrombosis (29). In patients with sepsis, nitric oxide (NO) and bacteria stimulate the production of platelet-derived exosomes. These exosomes can induce endothelial cell apoptosis by producing superoxide, NO, and peroxynitrite anions (30). It is also 


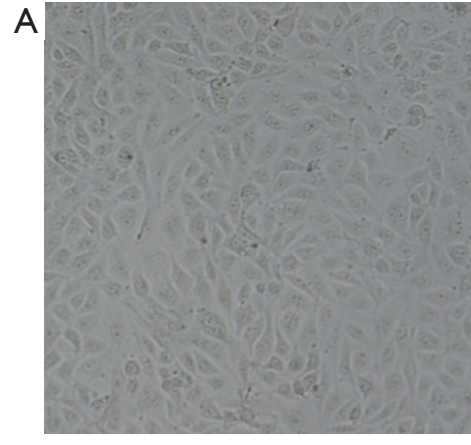

Control

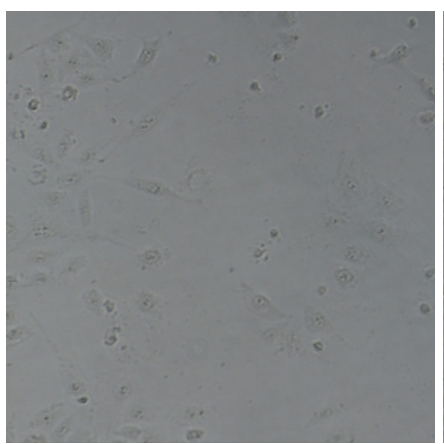

IVCL-3D

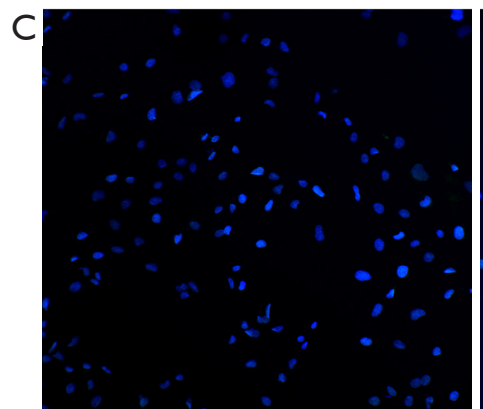

Control

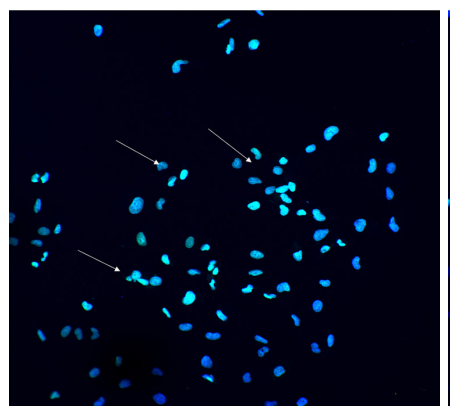

IVCL-3D

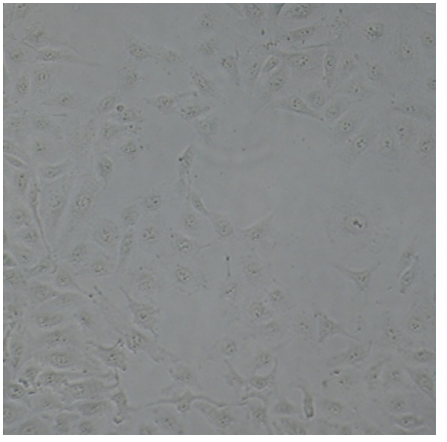

IVCL-1D

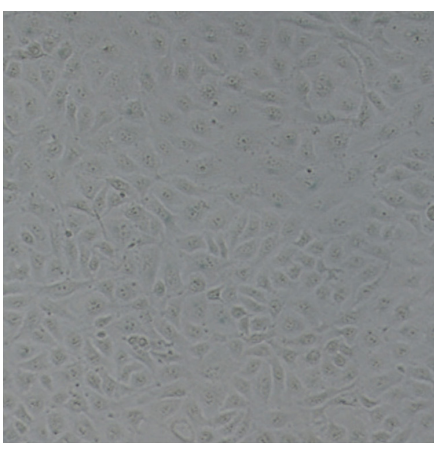

IVCL-7D

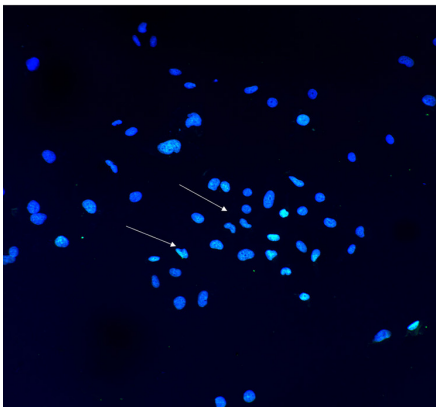

IVCL-1D

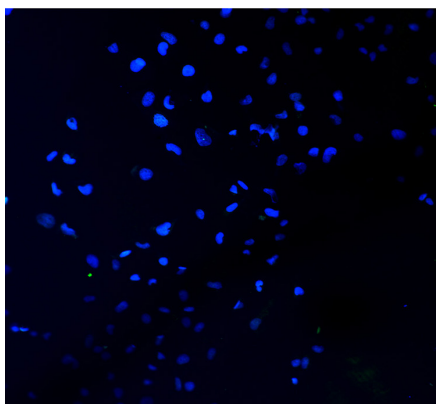

IVCL-7D
B

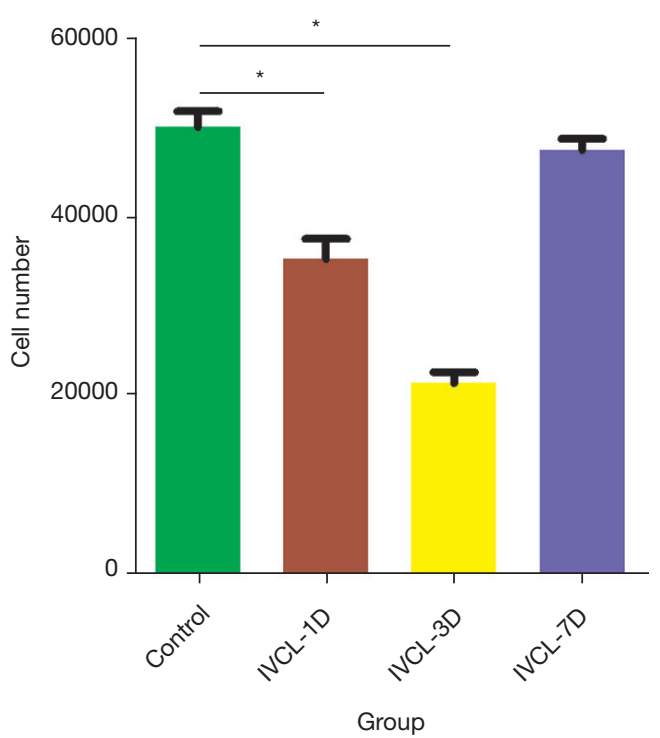

Figure 6 Exosomes containing MPO in the plasma of rats from different groups of thrombus models were co-cultured with HUVECs. (A) Relative growth status of HUVECs (IVCL-1D < IVCL-3D < IVCL-7D < control group) observed under a light microscope $(\times 100 \mu \mathrm{m})$. (B) CCK-8 assay showing the highest and lowest numbers of HUVECs in the control and IVCL-3D groups, respectively; data are presented as mean $\pm \mathrm{SD},{ }^{*}, \mathrm{P}<0.05$. (C) TUNEL staining of apoptosis of HUVECs: IVCL-3D > IVCL-1D > IVCL-7D $(\times 100 \mu \mathrm{m})$. The white arrow indicates apoptotic vascular endothelial cells. HUVECs, human umbilical vein endothelial cells; MPO, myeloperoxidase; CCK-8, Cell Counting Kit-8; TUNEL, terminal deoxynucleotidyl transferase dUTP nick end labeling. 
known that MPO plays an important role in promoting inflammation and oxidative stress and the results of the present study indicate that exosomal it plays a role in vascular endothelial cell damage.

\section{Conclusions}

During DVT formation, blood vessel walls are damaged by the release of plasma exosomes. We identified differences in exosomal MPO levels in orthopedic DVT and non-DVT patients. Thus, our results implicate exosomal MPO as a potential biomarker for early diagnosis of DVT. However, the molecular mechanism underlying the role of exosomal MPO in DVT remains to be elucidated.

\section{Acknowledgments}

Funding: This work was supported by the Guangdong Science and Technology Special Fund Mayor Project (20190301-65); Shantou City Science and Technology Plan Project, Guangdong Province, China (2018155, 2019-10637) and 2020 Li Ka Shing Foundation Cross-Disciplinary Research Grant (2020LKSFG01D).

\section{Footnote}

Reporting Checklist: The authors have completed the ARRIVE reporting checklist. Available at https://dx.doi. org/10.21037/atm-21-5583

Data Sharing Statement: Available at https://dx.doi. org/10.21037/atm-21-5583

Conflicts of Interest: All authors have completed the ICMJE uniform disclosure form (available at https://dx.doi. org/10.21037/atm-21-5583). The authors have no conflicts of interest to declare.

Ethical Statement: The authors are accountable for all aspects of the work in ensuring that questions related to the accuracy or integrity of any part of the work are appropriately investigated and resolved. All procedures performed in this study involving human participants were in accordance with the Declaration of Helsinki (as revised in 2013). The study was approved by the Ethics Committee of the Second Affiliated Hospital of Shantou University Medical College (China) (No. 2019-35) and informed consent was taken from all the patients. Experiments were performed under a project license (No. SUMC2019-244) granted by the Ethics Committee of the Second Affiliated Hospital of Shantou University Medical College (China), in compliance with guidelines of Experimental Animal Center of Medical College of Shantou University for the care and use of animals.

Open Access Statement: This is an Open Access article distributed in accordance with the Creative Commons Attribution-NonCommercial-NoDerivs 4.0 International License (CC BY-NC-ND 4.0), which permits the noncommercial replication and distribution of the article with the strict proviso that no changes or edits are made and the original work is properly cited (including links to both the formal publication through the relevant DOI and the license). See: https://creativecommons.org/licenses/by-nc-nd/4.0/.

\section{References}

1. Byrnes MC, Irwin E, Roach R, et al. Therapeutic anticoagulation can be safely accomplished in selected patients with traumatic intracranial hemorrhage. World J Emerg Surg 2012;7:25.

2. Chen TS, Lai RC, Lee MM, et al. Mesenchymal stem cell secretes microparticles enriched in pre-microRNAs. Nucleic Acids Res 2010;38:215-24.

3. Weber KT, Sun Y, Bhattacharya SK, et al. Myofibroblastmediated mechanisms of pathological remodelling of the heart. Nat Rev Cardiol 2013;10:15-26.

4. Ge Q, Zhou Y, Lu J, et al. miRNA in plasma exosome is stable under different storage conditions. Molecules 2014;19:1568-75.

5. Al Saleh HA, Haas-Neill S, Al-Hashimi A, et al. Thrombotic characteristics of extracellular vesicles derived from prostate cancer cells. Prostate 2018;78:953-61.

6. Kapustin AN, Shanahan CM. Emerging roles for vascular smooth muscle cell exosomes in calcification and coagulation. J Physiol 2016;594:2905-14.

7. Yu Y, Böing AN, Hau CM, et al. Tissue Factor Coagulant Activity is Regulated by the Plasma Membrane Microenvironment. Thromb Haemost 2018;118:990-1000.

8. Pearson JD. Endothelial cell function and thrombosis. Baillieres Clin Haematol 1994;7:441-52.

9. Sneddon AA, McLeod E, Wahle KW, et al. Cytokineinduced monocyte adhesion to endothelial cells involves platelet-activating factor: suppression by conjugated linoleic acid. Biochim Biophys Acta 2006;1761:793-801.

10. Yang J, Zhou X, Fan X, et al. mTORC1 promotes aging- 
related venous thrombosis in mice via elevation of platelet volume and activation. Blood 2016;128:615-24.

11. Fischer AH, Jacobson KA, Rose J, et al. Hematoxylin and eosin staining of tissue and cell sections. CSH Protoc 2008;2008:pdb.prot4986.

12. Blann AD, Lip GY. Virchow's triad revisited: the importance of soluble coagulation factors, the endothelium, and platelets. Thromb Res 2001;101:321-7.

13. Folsom AR, Lutsey PL, Heckbert SR, et al. Longitudinal increases in blood biomarkers of inflammation or cardiovascular disease and the incidence of venous thromboembolism. J Thromb Haemost 2018;16:1964-72.

14. Javanmard SH, Dana N. The effect of interferon $\gamma$ on endothelial cell nitric oxide production and apoptosis. Adv Biomed Res 2012;1:69.

15. Vandy FC, Stabler C, Eliassen AM, et al. Soluble $\mathrm{P}$-selectin for the diagnosis of lower extremity deep venous thrombosis. J Vasc Surg Venous Lymphat Disord 2013;1:117-25

16. Timp JF, Lijfering WM, Flinterman LE, et al. Predictive value of factor VIII levels for recurrent venous thrombosis: results from the MEGA follow-up study. J Thromb Haemost 2015;13:1823-32.

17. Kellermair J, Fellner A, Bittinger A, et al. Soluble platelet endothelial cell adhesion molecule 1 (sPECAM-1) improves diagnostic accuracy of D-Dimer in patients with suspected deep vein thrombosis (DVT). J Thromb Thrombolysis 2020;50:380-5.

18. Memon AA, Sundquist K, PirouziFard M, et al. Identification of novel diagnostic biomarkers for deep venous thrombosis. Br J Haematol 2018;181:378-85.

19. MacDougall DA, Feliu AL, Boccuzzi SJ, Lin J. Economic burden of deep-vein thrombosis, pulmonary embolism, and postthrombotic syndrome. Am J Health Syst Pharm 2006;63:S5-15.

20. Goldhaber SZ. Risk factors for venous thromboembolism. J Am Coll Cardiol 2010;56:1-7.

Cite this article as: Han Y, Bai X, Wang X. Exosomal myeloperoxidase as a biomarker of deep venous thrombosis. Ann Transl Med 2022;10(1):9. doi: 10.21037/atm-21-5583
21. Strom RG, Frempong-Boadu AK. Low-molecular-weight heparin prophylaxis 24 to 36 hours after degenerative spine surgery: risk of hemorrhage and venous thromboembolism. Spine (Phila Pa 1976) 2013;38:E1498-502.

22. Yang SD, Liu H, Sun YP, et al. Prevalence and risk factors of deep vein thrombosis in patients after spine surgery: a retrospective case-cohort study. Sci Rep 2015;5:11834.

23. Klebanoff SJ. Myeloperoxidase: friend and foe. J Leukoc Biol 2005;77:598-625.

24. Loria V, Dato I, Graziani F, et al. Myeloperoxidase: a new biomarker of inflammation in ischemic heart disease and acute coronary syndromes. Mediators Inflamm 2008;2008:135625.

25. Zhang Z, Hu L, Chen W, et al. Danhong huayu koufuye prevents deep vein thrombosis through anti-inflammation in rats. J Surg Res 2016;201:340-7.

26. Mullane KM, Kraemer R, Smith B. Myeloperoxidase activity as a quantitative assessment of neutrophil infiltration into ischemic myocardium. J Pharmacol Methods 1985;14:157-67.

27. Hansberry DR, Shah K, Agarwal P, et al. Fecal Myeloperoxidase as a Biomarker for Inflammatory Bowel Disease. Cureus 2017;9:e1004.

28. Ndrepepa G. Myeloperoxidase - A bridge linking inflammation and oxidative stress with cardiovascular disease. Clin Chim Acta 2019;493:36-51.

29. Kheansaard W, Phongpao K, Paiboonsukwong K, et al. Microparticles from $\beta$-thalassaemia/HbE patients induce endothelial cell dysfunction. Sci Rep 2018;8:13033.

30. Gambim MH, do Carmo Ade O, Marti L, et al. Plateletderived exosomes induce endothelial cell apoptosis through peroxynitrite generation: experimental evidence for a novel mechanism of septic vascular dysfunction. Crit Care 2007;11:R107.

(English Language Editor: J. Jones) 